\title{
Os benefícios da acupuntura no tratamento de Disfunção Da Articulação Temporomandibular (DTM): uma revisão da literatura
}

The benefits of acupuncture in the treatment of Temporomandibular Joint Dysfunction (TMD): a literature review

\section{Los beneficios de la acupuntura en el tratamiento de la Disfunción de la Articulación}

Temporomandibular (TMD): una revisión de la literatura

Recebido: 22/12/2021 | Revisado: 28/12/2021 | Aceito: 04/01/2022 | Publicado: 07/01/2022

\author{
Carlos Eduardo de Araújo Teles \\ ORCID: https://orcid.org/0000-0003-1631-1332 \\ Centro Universitário Mario Jucá Pontes, Brasil \\ E-mail: caduteeles@gmail.com \\ Yonara Barreto Tavares \\ ORCID: https://orcid.org/0000-0003-0253-0171 \\ Centro Universitário Mario Jucá Pontes, Brasil \\ E-mail: yonarabtavares@gmail.com \\ Alexandre Henrique Moura de Oliveira \\ ORCID: https://orcid.org/0000-0002-5374-9010 \\ Centro Universitário Mario Jucá Pontes, Brasil \\ E-mail: alexandre.oliveira@umj.edu.br
}

\begin{abstract}
Resumo
A disfunção da articulação temporomandibular (DTM) é um problema relacionado à saúde bucal que pode ter várias origens e formas de se manifestar no indivíduo. Muitos profissionais da saúde, e em especial da odontologia, tem utilizado a acupuntura no tratamento da DTM para reduzir a dor e a sua gravidade. Diante do exposto, a presente pesquisa tem por objetivo analisar a partir da revisão bibliográfica a importância da acupuntura no tratamento da Disfunção Temporomandibular. Quanto ao processo metodológico, adotou-se quanto ao objetivo, coleta de dados e análise dos resultados, respectivamente, a pesquisa descritiva, bibliográfica e análise de conteúdo. As produções científicas sobre a importância da acupuntura no tratamento da DTM revelam que essa técnica milenar chinesa tem a capacidade de reduzir a dor, relaxar os músculos e minimizar a gravidade da patologia. Conclui-se que a acupuntura é tão eficiente quanto outros procedimentos mais invasivos no tratamento da DTM, porém, ainda é pouco explorada pelos profissionais da saúde.
\end{abstract}

Palavras-chave: Acupuntura; Disfunção da articulação temporomandibular; Tratamento coadjuvante.

\begin{abstract}
Temporomandibular joint dysfunction (TMD) is a problem related to oral health that can have different origins and forms of manifestation in the individual. Many health professionals, especially dentists, have used acupuncture in the treatment of TMD to reduce pain and severity. Given the above, this research aims to analyze from the literature review the importance of acupuncture in the treatment of Temporomandibular Disorders. As for the methodological process, it was adopted as the objective, data collection and analysis of results, respectively, descriptive research, bibliographical and content analysis. Scientific productions on the importance of acupuncture in the treatment of TMD reveal that this ancient Chinese technique has the ability to reduce pain, relax muscles and minimize the severity of the pathology. It is concluded that acupuncture is as efficient as other more invasive procedures in the treatment of TMD, however, it is still little explored by health professionals.
\end{abstract}

Keywords: Acupuncture; Temporomandibular joint dysfunction; Adjuvant treatment.

\section{Resumen}

La disfunción de la articulación temporomandibular (DTM) es un problema relacionado con la salud bucal que puede tener diferentes orígenes y formas de manifestación en el individuo. Muchos profesionales de la salud, especialmente los dentistas, han utilizado la acupuntura en el tratamiento de TMD para reducir el dolor y su gravedad. Dado lo anterior, esta investigación tiene como objetivo analizar, a partir de la revisión de la literatura, la importancia de la acupuntura en el tratamiento de los Trastornos Temporomandibulares. En cuanto al proceso metodológico, se adoptó, en cuanto al objetivo, recolección de datos y análisis de resultados, respectivamente, investigación descriptiva, investigación bibliográfica y análisis de contenido. Producciones científicas sobre la importancia de la acupuntura en el tratamiento del TMD revelan que esta antigua técnica china tiene la capacidad de reducir el dolor, relajar los 
músculos y minimizar la gravedad de la patología. Se concluye que la acupuntura es tan eficaz como otros procedimientos más invasivos en el tratamiento de los TTM, sin embargo, aún es poco explorada por los profesionales de la salud.

Palabras clave: Acupuntura; Disfunción de la articulación temporomandibular; Tratamiento adyuvante.

\section{Introdução}

As políticas relacionadas à promoção de saúde bucal têm grande importância na conscientização da população de forma geral. O indivíduo estando em dia com consultas periódicas de seis em seis meses em média ou de acordo com seu cirurgião dentista, trará benefícios de forma bastante efetiva, prevenindo várias doenças, tais como cárie, gengivite, periodontite. Com a simples consulta de rotina pode se fazer um diagnóstico de uma outra doença pré-existente que o paciente ainda não tenha o conhecimento, como por exemplo a Disfunção Temporomandibular (DTM).

A antiga odontologia trouxe más experiências para a população, fazendo do ambiente odontológico um lugar de medo, desenvolvendo nos pacientes a odontofobia, termo que caracteriza por pessoas com medo/fobia de dentistas. $\mathrm{Na}$ tentativa de diminuir esse pânico e ansiedade, cirurgiões dentistas têm buscado desenvolver alternativas para driblar esses sinais clínicos do paciente. Trazer informações do tratamento que será executado de forma clara e objetiva para esse paciente é um dos primeiros passos além de outros, como um ambiente harmonioso, música, filmes, cadeiras massageadoras, aromaterapia e acupuntura.

Importante destacar que a ADTM é uma patologia que acomete as Articulações Temporomandibulares (ATMs), músculos mastigatórios e tecidos associados. De acordo com Neville, et al. (2016) o paciente pode apresentar sintomas como: dor ao mastigar, limitação de abertura da boca, estalos, zumbidos, dor nos dentes e na face. A dor é o principal gatilho para a procura de tratamento. Dentre as terapias não cirúrgicas, inclui-se a acupuntura, que é uma técnica milenar que faz parte da medicina tradicional chinesa.

Diante do exposto, a presente pesquisa tem por objetivo analisar a partir da revisão bibliográfica a importância da acupuntura no tratamento da Disfunção Temporomandibular (DTM). Nesse sentido, trata-se de uma pesquisa descritiva quanto ao objetivo, bibliográfica quanto a coleta de dados, numa abordagem qualitativa, e análise de conteúdo quanto aos resultados.

Como pondera Gil (2008) diz-se que uma pesquisa é descritiva quando o pesquisador busca descrever todos os elementos pertencentes ao objeto de estudo, bem como a forma como ele interage no ambiente. Dessa forma, buscou-se descrever como os pesquisadores têm discutido a importância e resultados da acupuntura no processo de tratamento e redução da gravidade da DTM.

De acordo com Lakatos e Marconi (2021), a pesquisa bibliográfica refere-se a uma técnica metodológica utilizada por pesquisadores para proporcionar o máximo de informações acerca de determinada temática em produções científicas que receberam algum tipo de tratamento analítico, como artigos científicos, livros, capítulos, dentre outros. Assim, foi realizada uma pesquisa nas bases de dados Literatura Latino-Americana e do Caribe em Ciências da Saúde (LILACS), Biblioteca Virtual de Saúde (BVS Brasil), Scientific Electronic Library Online (SCIELO Brasil) e o Google Acadêmico para realizar um levantamento de trabalhos científicos que tratassem da acupuntura e da DTM. Assim, foram escolhidos os descritores: Acupuntura e DTM, Acupuntura e Disfunção Temporomandibular, Acupuntura e odontologia, Acupuntura e benefícios.

Conforme preconiza Bardin (2011), a análise de conteúdo é um método que busca analisar documentos de forma qualitativa, a fim de proporcionar ao pesquisador novas descobertas sobre determinados assuntos. Importante destacar que, análise das produções deu-se de forma não apriorística, ou seja, sem quaisquer categorizações, na medida que foram sendo identificadas, foram sendo interpretadas e organizadas cronologicamente.

A pesquisa é composta estruturalmente por esta introdução, a qual introduz aspectos relacionados a DTM e a importância da acupuntura no processo de redução da dor e da gravidade da patologia, o objetivo e o processo metodológico; 
uma seção que versa sobre o que é a DTM, suas causas e possíveis tratamentos; uma seção que trata sobre os benefícios da acupuntura no tratamento da DTM, apresentando também como os pesquisadores brasileiros demonstram a eficiência desse tratamento humanístico e não invasivo; e por fim, a conclusão com as constatações principais sobre a temática em estudo.

\section{Disfunção Temporomandibular (DTM): Causas e Tratamentos}

De acordo com Donnarumma, et al. (2010), o termo Disfunção Temporomandibular (DTM) compreende um conjunto de doenças que podem acometer os músculos mastigatórios e outros presentes na face humana. Ainda conforme os respectivos autores, não há com exatidão uma motivação para o surgimento da patologia, sendo considerada como multifatorial. Dentre as principais causas estão:

[...] fatores estruturais, neuromusculares, oclusais 11 (perdas dentárias, desgaste dental, próteses mal adaptadas, cáries, restaurações inadequadas „INTRODUÇÃO A articulação temporomandibular (ATM) é a única articulação móvel do crânio. É considerada a mais complexa do corpo humano, por duas razões: é a única que permite movimentos rotacionais e translacionais, devido à articulação dupla do côndilo. DTM: sinais e atuação multidisciplinar entre outras), psicológicos (devido a tensão há um aumento da atividade muscular que gera espasmo e fadiga), hábitos parafuncionais (bruxismo, onicofagia, apoio de mão na mandíbula, sucção digital ou de chupeta) 2 e lesões traumáticas ou degenerativas da ATM 1. (Donnarumma, 2010, p. 788-789).

A DTM é classificada em Muscular ou Artrogênica, e os tratamentos envolvem terapias cirúrgicas e não cirúrgicas. A DTM acomete cerca de $33 \%$ da população adulta, onde as mulheres de meia idade são mais comumente acometidas por essa disfunção, mas pode afetar pessoas de ambos os sexos e de qualquer idade, e cerca de 15,6\% têm necessidade de tratamento. Investiga-se uma mudança expressiva desse padrão nos últimos anos, com a eclosão de novos casos em indivíduos com menores idades e com a progressão de pessoas do sexo masculino abalados por ela, de acordo com dados da The American Academy of Orofacial Pain.

Sua etiologia ainda é desconhecida, mas está associada a uma variedade de condições que reduzem a capacidade adaptativa das ATMs, a classificação até o momento se dividem de acordo com sua possível origem:

- Muscular - Com acometimento dos músculos da mastigação (Masseter, Temporal, Pterigóideo medial e Pterigóideo lateral);

- Articular - Com acometimento das estruturas próprias da ATM (Cápsula, Disco, Ligamentos e Membrana Sinovial);

- Muscular e Articular - Quando acomete ambos os componentes.

Uma das principais causas da DTM são através das colisões, onde dois ou mais corpos exercem forças um sobre o outro por um tempo relativamente considerável e curto, como por exemplo, ao dormir o paciente, inconscientemente, exerce força excessiva ao fechar a boca, apertando os dentes uns contra os outros, ou por meio de um choque físico de grande magnitude, mais comum em esportistas, onde o contato físico é constante e muitas das vezes agressivo, ou simplesmente através do hábito de roer unhas. Outras causas importantes são: alterações do estado emocional, fatores genéticos e hábitos orais, como por exemplo ações instintivas ao sentir raiva e ansiedade, ou mastigação incorreta.

Os primeiros sinais são a sensibilidade à palpação nos músculos da mastigação e da ATM, junto a limitação ou incoordenação dos movimentos mandibulares e ruídos articulares. Os sintomas mais comuns são dores na face, dores na cabeça e no ouvido, vertigem, zumbido e plenitude auricular.

O diagnóstico tem seus critérios baseados no Diagnostic Criteria (DC), método baseado por uma série de questionamentos que precisam ser feitos ao paciente, com o intuito de compreender se de fato trata-se de uma DTM ou outra 
patologia que tenha sintomas similares. (Schiffman, et al. 2014). Assim, Pereira Júnior e Gonçalves (2014) compreendem que esse método perpassa por questões como perguntas sobre dores na mandíbula, de cabeça e de ouvido, para entender se a dor é recente ou anterior, contatar o grau da dor, e qual o local específico dela; se há ruídos ao movimentar a mandíbula ou travamentos; dentre outros sintomas. Realizada a fase de questionamentos, passa-se para a etapa de procedimentos de exames e comandos de abertura da boca, movimento do queixo e palpação nos músculos, devendo o profissional registrar qualquer informação sobre dores e desconfortos apresentada pelo paciente.

E os tratamentos para a disfunção tem como objetivo a redução dos níveis de dor, restaurar a função do sistema mastigatório e promover uma melhora na qualidade de vida do paciente. Os tratamentos envolvem terapias não cirúrgicas, como por exemplo: sessões de fisioterapia, Massagem, Termoterapia (Aplicação terapêutica de calor ou frio no local, para estimular a vasodilatação ou vasoconstrição), Terapia medicamentosa (Fazendo o uso de Dipirona Sódica 500mg e Paracetamol 500mg, ou Ibuprofeno 600mg de $8 \mathrm{em} 8$ horas em caso de dores musculares e articulares.), Abordagem psicoterapêutica (Quando se identifica a necessidade de apoio psicológico, em quadros que estão associados a ansiedade e depressão), exercícios terapêuticos (Contribuem para a restauração da amplitude de movimento), e uso noturno da placa oclusal, feita sob medida para o paciente. E os procedimentos cirúrgicos, quando o paciente apresenta alterações em partes da mandíbula, e os tratamentos anteriores não surtirem efeito.

Os tratamentos cirúrgicos são designados apenas para cerca de $2 \%$ dos pacientes, em casos de exceção, especificamente quando ocorrem alterações internas na articulação, como por exemplo, o deslocamento do disco articular que é o resultado do choque físico, como por exemplo, ao apertar excessivamente os dentes ou receber um golpe com grande intensidade na região. Em pacientes que têm diagnósticos de depressão, bruxismo noturno e assintomáticos a cirurgia deve ser evitada. As modalidades terapêuticas empregadas na cirurgia da DTM são: Manipulação mandibular assistida com aumento de pressão hidrostática (MMAAPH); Artrocentese; Artroscopia e Artrotomia, que pode ser subdividida em ancoragem do disco, discectomia com ou sem interposição de material, Tuberculotomia, Condilectomia com enxerto, substituição total articular e reposicionamento discal.

\section{Os benefícios da acupuntura no tratamento da DTM}

Atualmente é possível observar tratamentos de última geração, com tecnologia avançada para a grande maioria dos procedimentos odontológicos, porém a grande descoberta para o auxílio no tratamento da DTM é uma técnica milenar oriental, a acupuntura, que assegura a eficácia no tratamento de diversas doenças, como patologias do sistema musculoesquelético, doenças neurológicas, doenças respiratórias, patologias gastrointestinais e enfermidades orofaciais, e tem se mostrado um caminho promissor para o profissional e paciente.

De acordo com Florian, Meirelles e Sousa (2011, p. 190),

Como uma filosofia a, ciência e terapia holística, prega o equilíbrio físico, mental e emocional através do equilíbrio do fluxo energético dos meridianos, que são uma teia de canais de energia que percorrem todo o corpo e dos órgãos e vísceras internos (Zang Fu), que comandam o funcionamento do organismo.

Vários estudos e ensaios clínicos demonstraram a eficácia da acupuntura no tratamento da dor de modo geral, e na odontologia tem sido eficaz no tratamento de dores orofaciais como neuralgias trigeminais idiopáticas, herpes-zoster, sinusites maxilares, dores dentais, artrose da ATM, bem como em pacientes portadores xerostomia, bruxismo e DTM, além do controle e diminuição significante nas comorbidades associadas como ansiedade e odontofobia.

Importante destacar que, como observado por Yamamura (2004, p. 76), as agulhas na acupuntura, 
[...] após atravessar a pele e a tela subcutânea, penetra o primeiro músculo interósseo do dorso e atinge o músculo indutor do polegar. Superficialmente, a agulha relaciona-se com os nervos digitais dorsais do ramo superficial do nervo radial e com os nervos digitais palmares próprios do nervo mediano. Profundamente, relaciona-se com o ramo profundo do nervo ulnar.

Nesse sentido, a técnica da acupuntura não se trata de uma inserção desordenada de agulhas sob a pele do paciente, requer uma série de técnicas para identificar os canais meridianos de energia, e como estes reagem no sistema nervoso do indivíduo.

As DTMs são similares a outras disfunções reumatológicas e musculoesqueléticas e, em razão do pouco conhecimento sobre o curso natural dessa alteração, um esforço especial deve ser feito para evitar terapias mais agressivas e irreversíveis, e a acupuntura se encaixa perfeitamente nesses parâmetros, e seu mecanismo de ação do ponto de vista neuroanatômico, mostra como a acupuntura funciona para a analgesia, devido a DTM se tratar de uma patologia de causa multifatorial, neste caso específico, a inserção das agulhas provoca a liberação de neurotransmissores, como endorfina e serotonina, isso gera uma redução e remissão da dor quando comparados por exemplo, ao uso de placa oclusal. Ela tem efeitos anti-inflamatórios, ansiolíticos, miorrelaxantes e ativador da função imunológica.

Os benefícios surgem em médio-longo prazo, apesar de algumas declarações de pacientes que sinalizaram o alívio imediato da dor após a primeira sessão, por ser um método com baixo índice de efeitos colaterais, barato, não invasivo e reversível, seu uso pode ser contínuo e sem contra indicações, podendo auxiliar o profissional no tratamento da DTM, com risco quase nulo, tratando também fatores coadjuvantes da DTM.

Esse tratamento intervém sobre os aspectos físicos do paciente, pela liberação de opióides e melhora do fluxo sanguíneo, e psicológicos, capaz de agir no relaxamento emocional do indivíduo. O que leva à divisão de opiniões dentro da literatura, embora não exista um protocolo padrão sobre pontos de aplicações e quantidade de sessões necessárias para esse tratamento, ter uma visão holística sobre o caso e o paciente é indispensável, pois a análise do caso deve ver o paciente comum todo e não com um panorama fragmentado sobre a região afetada.

Quadro 1 - trabalhos relacionados a acupuntura e DTM nas bases de dados.

\begin{tabular}{|c|c|c|}
\hline Ano de publicação & Autores (as) & Principais discussões \\
\hline 2005 & $\begin{array}{l}\text { Branco, Fonseca, Oliveira, Gomes } \\
\text { e Neto. }\end{array}$ & $\begin{array}{l}\text { Apresentam a importância da acupuntura para o tratamento da } \\
\text { DTM, e mais especificamente para o relaxamento muscular e } \\
\text { redução da dor. }\end{array}$ \\
\hline 2010 & Zotelli, Meirelles Sousa. & $\begin{array}{l}\text { Publicação de resultados clínicos de inserção da acupuntura no } \\
\text { tratamento de DTM, como também sementes para a } \\
\text { auriculoterapia. }\end{array}$ \\
\hline 2011 & Florian, Meirelles e Sousa & $\begin{array}{l}\text { Nesta pesquisa são discutidas as possíveis formas de } \\
\text { desenvolvimento da DTM, como também apresenta resultados de } \\
\text { estudo clínico. }\end{array}$ \\
\hline 2011 & Borin , Corrêa, Silva e Milanesi. & $\begin{array}{l}\text { Nesta pesquisa, também foi realizado um estudo de caso, com } \\
\text { uma amostra de } 40 \text { mulheres de idade entre } 20 \text { e } 40 \text { anos, } \\
\text { diagnosticadas com DTM, a fim de averiguar a escala de dor e a } \\
\text { gravidade da patologia, utilizando para a redução dos problemas } \\
\text { a acupuntura. }\end{array}$ \\
\hline 2012 & $\begin{array}{l}\text { Itoh, Asai, Ohyabu, Imai e } \\
\text { Kitakoji. }\end{array}$ & $\begin{array}{l}\text { Traz importantes contribuições para novos estudos sobre a } \\
\text { acupuntura no tratamento da DTM. Os resultados apontaram para } \\
\text { uma redução e alívio da dor, confirmando assim que a técnica } \\
\text { milenar chinesa tem capacidade de ser mais explorada para os } \\
\text { tratamentos de determinadas enfermidades. }\end{array}$ \\
\hline 2016 & $\begin{array}{l}\text { Garbelotti, Turci, Serigato, Pizzol e } \\
\text { Franco-Micheloni. }\end{array}$ & $\begin{array}{l}\text { Apresenta resultados de estudo clínico com pacientes portadores } \\
\text { de DTM, utilizando a acupuntura como técnica menos invasiva e } \\
\text { eficaz. }\end{array}$ \\
\hline 2017 & $\begin{array}{l}\text { Ferreira, Grossmann, Januzzi, } \\
\text { Gonçalves, Mares, Queiroz de } \\
\text { Paula e Carvalho. }\end{array}$ & $\begin{array}{l}\text { Trata-se da apresentação dos resultados de um ensaio clínico } \\
\text { controlado no laboratório da Universidade Federal de Juiz de } \\
\text { Fora, no qual foram selecionadas } 20 \text { mulheres meses antes, as }\end{array}$ \\
\hline
\end{tabular}




\begin{tabular}{|c|l|l|}
\hline & & quais foram diagnosticadas com DTM \\
\hline 2018 & Freire, Freire e Dias-Ribeiro. & $\begin{array}{l}\text { Trata-se de um estudo clínico, no qual evidenciou-se os } \\
\text { resultados da acupuntura para o tratamento de dores orofaciais, } \\
\text { enquanto método complementar. }\end{array}$ \\
\hline 2018 & $\begin{array}{l}\text { Matias, Ribeiro, Dallanora, } \\
\text { Comunello, Wesoloski, Amaral e } \\
\text { Dallanora, }\end{array}$ & $\begin{array}{l}\text { Neste trabalho, os referidos autores também exploraram a } \\
\text { importância da acupuntura no tratamento dessa doença que } \\
\text { acomete diversos indivíduos na atualidade. }\end{array}$ \\
\hline 2019 & $\begin{array}{l}\text { Oscaine, Pontes, Castillo, Suliano, } \\
\text { Oshiro Filho. }\end{array}$ & $\begin{array}{l}\text { Estudo clínico com 34 pacientes portadores de DTM, realizado } \\
\text { na Universidade do Mato Grosso do Sul, utilizando a acupuntura } \\
\text { como tratamento de redução da dor. }\end{array}$ \\
\hline 2020 & $\begin{array}{l}\text { Aguiar, Lima, Freitas, Pinheiro, } \\
\text { Souza, Caldas Júnior e Melo. } \\
\text { quais, 25 foram tratados com acupuntura, e 25 com métodos } \\
\text { convencionais de tratamento da dor oriunda da DTM. }\end{array}$ \\
\hline
\end{tabular}

Fonte: Elaborado pelos autores (2021).

Em 2005, os pesquisadores Carolina Assaf Branco, Rodrigo Borges Fonseca, Terezinha Rezende Carvalho de Oliveira, Vanderlei Luiz Gomes, Alfredo Júlio Fernandes Neto, desenvolveram o artigo intitulado "Acupuntura como tratamento complementar nas disfunções temporomandibulares: revisão da literatura", o qual foi publicado na Revista de Odontologia da UNESP. Preliminarmente, os autores fizeram algumas alegações acerca de dores faciais e sobre como um profissional da saúde pode dar um diagnóstico errado para o paciente, o que pode resultar em procedimentos invasivos, bem como perda de dentes saudáveis, interferindo assim na qualidade de vida do indivíduo.

No tocante a DTM, Branco, et al. (2005, p. 14) afirmam que esta pode ser considerada como "[...] o conjunto de anormalidades responsáveis por dores crônicas do tipo recorrente, não progressivas e associadas a impacto leve ou moderado na atividade social do paciente". Existem alguns métodos utilizados na odontologia para o tratamento desse problema, sendo os principais deles o uso de placa oclusal, sendo considerado pelos autores um dos mais eficientes nos casos de DTM muscular, pois "[...] reduz a hiperatividade muscular por meio de mudanças periféricas originadas da alteração do impulso aferente nos receptores orgânicos, conduzindo a uma resposta eferente que reduz a tensão muscular da região em questão."

Em relação a acupuntura no tratamento da DTM, os autores refletem que esta se mostra também como uma técnica eficiente no processo de controle da dor, relaxamento muscular, uma vez que as agulhas promovem a liberação de endorfina. Outrossim, os autores também apresentam que, tanto a acupuntura quanto os procedimentos tradicionais demonstram resultados semelhantes no processo de alívio e controle da dor de indivíduos portadores da DTM. E mais especificamente sobre a acupuntura, Branco, et al. (2005, p. 15) concluem que "[...] essa técnica utiliza o mecanismo de analgesia próprio do organismo sem provocar quaisquer efeitos colaterais, podendo ser usada quantas vezes forem necessárias."

No ano de 2010, as autoras Vera Lucia Rasera Zotelli, Maria Paula Maciel Rando Meirelles e Maria da Luz Rosário de Sousa publicaram na Revista de Odontologia da Universidade Cidade de São Paulo o artigo intitulado Uso da acupuntura no manejo da dor em pacientes com alterações na articulação temporomandibular (ATM). A pesquisa foi fruto de um estudo de caso prático, para conhecer como a técnica milenar da acupuntura pode ser útil no tratamento de distúrbios que podem ocorrer na Articulação Temporomandibular (ATM), uma vez que, na concepção dos especialistas em acupuntura, "[...] a doença é resultado da interação entre os agentes agressores e a resposta do organismo, comandada pelo sistema nervoso central." (Zotelli et al., 2010, p. 186).

Logo, a acupuntura oferece uma série de benefícios para os pacientes portadores da DTM, uma vez que essa técnica alcança o sistema nervoso, possibilitando assim uma melhor qualidade de vida. Entretanto, é necessário que o profissional ao inserir a acupuntura no tratamento dessa doença possua conhecimento sobre quais pontos devem ser estimulados, como ocorre o processo da circulação do sangue, para que assim seja possível a promoção do relaxamento muscular, redução da inflamação, e também da dor. 
De acordo com Zotelli, Meirelles e Sousa (2010), o uso da acupuntura deve ser realizado após o correto diagnóstico, não devendo ser aplicada de forma genérica para qualquer paciente. Logo, é importante reconhecer as particularidades de cada indivíduo, e verificar se a técnica da acupuntura pode ser incluída como tratamento coadjuvante.

Em relação à aplicação da acupuntura no caso concreto, as autoras relaram que o procedimento ocorreu com uma paciente do sexo feminino, no Serviço Odontológico da Faculdade de Odontologia de Piracicaba (FOP-UNICAMP), a qual procurou pelos profissionais de odontologia reclamando de dores na ATM. Após o pré-atendimento, iniciou-se a aplicação de agulhas, anamnese e os exames de língua e pulso. No processo de atendimento, a paciente apresentou alguns problemas pelos quais estava passando, como medo de dirigir, síndrome do pânico e distúrbio do sono. Conforme relatado na pesquisa, Zotelli, Meirelles e Sousa (2010, p. 187):

$\mathrm{Na}$ anamnese segundo a MTC, a paciente relatou preferir calor, ter transpiração excessiva no tórax e na cabeça, sentir muita sede e beber mais refrigerantes do que água; preferir alimentos salgados, porém, sente muita necessidade de chocolate; ter sono leve; ter grande produção de cera no ouvido; retenção de líquidos; urina pouca e de cor escura e com odor forte; ser obstipada. A tez era amarelada, oleosa e porosa com cicatrizes de acne, manchas e sardas; a língua possuía marcas de dentes nas laterais, saburra amarela no centro e ponta avermelhada; pulso médio, rápido e escorregadio, lábios e gengivas normais. Declarou-se ser ansiosa, perfeccionista, preocupada e imediatista.

Diante dos dados apresentados, concluiu-se a partir da Medicina Tradicional Chinesa e dos sintomas de insônia, preocupação, palidez, oleosidade da tez, dentre outros, a paciente possuía deficiência de Yang do Baço-pâncreas. Na sessão posterior, já com o diagnóstico individualizado, utilizou-se a acupuntura sistêmica e auricular, em sessões de vinte minutos, agulhas descartáveis de calibre $0.25 \times 25 \mathrm{~mm}$ para as punções, como também sementes para a auriculoterapia. "Após as sessões, a paciente observou melhora da dor na região da articulação e relatou também ter tido mais coragem para enfrentar o seu medo de dirigir seu veículo." (Zotelli et al., 2010, p. 187).

Em 2011, foi publicado o trabalho das pesquisadoras Marcelo Rossiti Florian, Maria Paula Maciel Rando Meirelles e Maria da Luz Rosário de Sousa, denominado "Disfunção temporomandibular e acupuntura: uma terapia integrativa e complementar". Assim como os trabalhos apresentados anteriormente, os autores definiram a DTM, refletiram sobre as múltiplas causas do seu surgimento, apresentaram também quais são os tratamentos existentes e como a acupuntura pode ser útil nesse tratamento.

Os autores chamam atenção para a forma de diagnosticar o paciente com DTM, uma vez que existem outros problemas de saúde que apresentam características similares. Nesse sentido,

Seu diagnóstico é algo que deve ser feito cuidadosa e criteriosamente, pois são muitas as doenças e disfunções que originam sintomas semelhantes, tais como: problemas de origem odontogênica, cefaleia tensional, enxaquecas, fibromialgia, sinusite, distúrbios de glândulas salivares, neuralgias, problemas cervicais, problemas otológicos e artrites, dentre outras. (Florian; Meirelles; Sousa, 2011, p. 189-190).

Para a realização do tratamento, os profissionais da saúde odontológica têm recorrido a tratamentos menos invasivos, privilegiando assim a qualidade de vida do indivíduo. Dessa forma, os autores citam como exemplos desses tratamentos o uso de plantas oclusais, fisioterapia local com o uso de calor, frio e exercícios, medicamentos para redução da dor e inflamação, compressão, e também a acupuntura. Em relação a esse último, Florian, Meirelles e Sousa (2011, p. 190) aludem que "A acupuntura tem sido incluída nesse rol de tratamentos não invasivos e reversíveis, por ser uma terapia segura, altamente individualizada ao paciente, que pode agir tanto localmente na remissão e no controle dos sintomas locais quanto no fator estresse emocional." 
O trabalho em questão também é fruto de um estudo clínico, o qual ocorreu com uma paciente de 32 anos, a qual apresentou os seguintes sintomas:

[...] relatando duas crises recentes de DTM, com intervalo de 2 meses entre elas. Essas crises afetaram o lado direito, com dor na região de ATM, masseter e temporal, EVA 6 e perduraram por aproximadamente 15 dias cada. As dores, segundo relato da paciente, eram do tipo pontada e persistente que aumentavam com pressão manual. Ela relatou não se lembrar de ter tido experiência desse tipo de problema antes desses episódios. (FLORIAN; MEIRELLES; SOUSA, 2011, p. 189-190).

Além desses sintomas, foram informadas pela paciente outras situações que podem ter influência nas dores apresentadas na ATM, como cefaléia, utilização de paracetamol, crise de otite, sinusite, bronquite, tosse seca, rinite e alergias. Seguindo os procedimentos da MTC, a paciente também informou que estava passando por problemas emocionais, devido ao processo de desenvolvimento de dissertação de mestrado, ocasionando também em redução do sono.

Após o primeiro atendimento, os profissionais explicaram para a paciente sobre o que é a DTM, bem como sobre a acupuntura no processo de tratamento. Além disso, realizou-se "[...] aconselhamento preventivo, relaxamento e exercícios respiratórios e auriculoterapia, com protocolo de pontos para DTM (pontos Shenmen, neurastenia, coração e maxilar-DTM)." (Florian; Meirelles; Sousa, 2011, p. 189-190).

No total, foram realizadas cinco sessões, sendo a primeira para a investigação e diagnóstico do problema. Na segunda, a paciente "[...] relatou que estava sem dor muscular, mas com cefaleia e dor dentro do ouvido esquerdo. Relatou, também, ter ficado muito tempo em sala com ar condicionado regulado em temperatura muito baixa." Importante destacar que, no tocante aos pontos de acupuntura "[...] utilizados foram R7, F2, VB34+TA17, VB20, VB39, protocolo para o padrão definido. Quando foi agulhado o ponto VB20, a paciente relatou cessamento imediato da cefaleia e da dor no ouvido." (Florian; Meirelles; Sousa, 2011, p. 189-190).

Dessarte, na visão dos pesquisadores,

A acupuntura tem, na prática, se mostrado bastante eficaz em casos de problemas musculares com ou sem envolvimento de origem emocional 5,10. Apesar de parecer ao raciocínio cartesiano ocidental uma ciência, até certo ponto, incompreensível e dúbia, a acupuntura é realizada sob uma vasta e experimentada teoria filosófica e médica 8,9, que, através de uma investigação muito bem acurada, procura formular diagnóstico e um plano de tratamento individualizado e bem definido. (Florian et al., 2011, p. 191).

A partir desse estudo, os autores vislumbram o poder que a acupuntura possui no processo de tratamento da DTM, sem a necessidade de se aderir a procedimentos invasivos e de certa forma prejudiciais à saúde do paciente.

Também no ano de 2011, foi publicado na revista Fisioterapia e Pesquisa o artigo "Acupuntura como recurso terapêutico na dor e na gravidade da desordem temporomandibular", de autoria de Graciele da Silva Borin, Eliane Castilhos Corrêa, Ana Maria Toniolo da Silva e Jovana Moura Milanesi. Nesta pesquisa, também foi realizado um estudo de caso, com uma amostra de 40 mulheres de idade entre 20 e 40 anos, diagnosticadas com DTM, a fim de averiguar a escala de dor e a gravidade da patologia, utilizando para a redução dos problemas a acupuntura.

O tratamento foi realizado com agulhas descartáveis $(0,25 \mathrm{X} 0,15 \mathrm{~mm})$ inseridas nos respectivos pontos com a pele previamente limpa com algodão e álcool etílico 70\%. Durante a aplicação da terapia, cada participante permaneceu completamente relaxada, deitada em uma maca na posição de decúbito dorsal por 30 minutos. A terapia de acupuntura totalizou dez atendimentos. (Borin, et al., 2011, p. 218). 
Dentre os principais resultados, foi constatado que "[...] antes do tratamento os indivíduos apresentaram a seguinte classificação para DTM: 6 com grau moderado e 14 com grau severo. Após o tratamento, observou-se por esta classificação: 7 com grau leve, 10 com grau moderado e 3 com grau grave.” (Borin, et al., 2011, p. 219).

Os relatos das pacientes e os dados obtidos na pesquisa revelam que houve redução da dor e da gravidade da DTM. Uma das possíveis respostas para a redução da dor é que "A acupuntura é um recurso terapêutico que intervém sobre os aspectos psicológicos e físicos do paciente, pela liberação de opioides e melhora do fluxo sanguíneo, respectivamente, o que pode ter contribuído para o alívio da dor dos pacientes deste estudo.” (Borin, et al., 2011, p. 220).

Dessarte, para as pesquisadoras, a acupuntura tem demonstrado resultados positivos no tratamento e redução da gravidade da DTM, podendo ser inserida também em outros tratamentos para este fim.

Apesar de ser de 2012, o estudo dos pesquisadores Kazunori Itoh, Sayo Asai, Hideaki Ohyabu, Kenji Imai e Hiroshi Kitakoji denominado "Effects of trigger point acupuncture treatment on temporomandibular disorders: a preliminary randomized clinical trial", publicado pela Journal of Acupuncture and Meridian Studies, traz em seu bojo importantes contribuições para novos estudos sobre a acupuntura no tratamento da DTM. Trata-se de um estudo clínico, realizado em 10 semanas com 16 pacientes, com um total de cinco sessões de acupuntura. Os resultados apontaram para uma redução e alívio da dor, confirmando assim que a técnica milenar chinesa tem capacidade de ser mais explorada para os tratamentos de determinadas enfermidades.

Em 2015, os pesquisadores Luciano Ambrosio Ferreira, Eduardo Grossmann, Eduardo Januzzi, Rafael Tardin Rosa Ferraz Gonçalves, Fernando Antonio Guedes Mares, Marcos Vinicius Queiroz de Paula e Antonio Carlos Pires Carvalho publicaram no periódico Hindawi Publishing Corporation o estudo intitulado "Ear Acupuncture Therapy for Masticatory Myofascial and Temporomandibular Pain: A Controlled Clinical Trial”. Trata-se da apresentação dos resultados de um ensaio clínico controlado no laboratório da Universidade Federal de Juiz de Fora, no qual foram selecionadas 20 mulheres meses antes, as quais foram diagnosticadas com DTM. Essas mulheres foram divididas em dois grandes grupos, sendo um de estudo, utilizando a acupuntura auricular associada a placa oclusal e um grupo controle com uso da placa de placa oclusal apenas.

No fim do experimento, os pesquisadores constataram que os dois grupos submetidos aos tratamentos diferentes tiveram redução da dor no decorrer das sessões, porém, estatisticamente, o grupo de estudo na primeira semana de terapia apresentou resultados significativos.

Outro trabalho importante sobre os efeitos da acupuntura identificado na coleta de dados foi o dos autores Vera Zotelli, Cássia Grillo, Maria Gil, Ronaldo Wada, Jorge Sato, Maria da Luz Rosário de Sousa, intitulado "Acupuncture Effect on Pain, Mouth Opening Limitation and on the Energy Meridians in Patients with Temporomandibular Dysfunction: A Randomized Controlled Trial", publicado no periódico Journal of Acupuncture and Meridian Studies, em 2017. Nessa pesquisa, os autores buscaram definir que a inclusão da MTC é importante para identificar os pontos meridianos, ou seja, os canais que emanam energia no corpo, que quando encontrados e perfurados no processo de acupuntura possibilita a reorganização da energia, inibindo o surgimento de diversas doenças. O método utilizado para medir a energia nos meridianos baseia-se em um desenvolvido pelo doutor japonês Nakatami, denominado de Ryodoraku.

Apesar de o trabalho ter sido publicado em 2017, a pesquisa foi realizada entre 2015 e 2016, na Clínica de Especialização da Faculdade de Odontologia de Piracicaba. O procedimento de escolha para a amostra deu-se com indivíduos com DTM, tanto muscular como mista, entre 20 e 50 anos, excluídos mulheres grávidas, pessoas com medo de agulha e quem estava fazendo outro tratamento. Assim, 43 pacientes foram divididos em dois grupos, um com tratamento de acupuntura real com placebo e outro com acupuntura simulada não penetrante. Para avaliar a dor, utilizou-se a escala numérica analógica (NVAS), com variação 0 (sem dor) e 10 (muita dor). A energia foi avaliada a partir do método Ryodoraku, representando 12 meridianos de acupuntura, os quais ficam localizados nos pulsos da mão direita e esquerda: pulmão (LU9-Taiyuan), pericárdio 
(PC7-Daling), coração (HT7-Shemen), intestino delgado (SI5-Yanggu), triplo energizador (TE4-Yangchi), intestino grosso (LI5-Yangxi) e 12 outros, localizados nos pés direito e esquerdo: baço (SP3-Taibai), fígado (F3-Taichong), rim (KI3-Taixi), bexiga (BL64-Jinggu), vesícula bexiga (GB40-Qiuxu) e estômago (ST42-Chongyang).

Os pacientes foram acompanhados em quatro sessões pelos pesquisadores, e no término, observou-se que houve significativa redução da dor, maior abertura da boca sem incômodos em ambos os grupos já em relação a energia nos meridianos, notou-se que no grupo com acupuntura real, a energia diminuiu durante as sessões, enquanto no segundo grupo manteve-se imutável.

No ano de 2018, os pesquisadores Bruna Joana Matias, Josiani Zonta Castegnaro Ribeiro, Lea Maria Dallanora, Rodrigo Cecconello, Soraia Hack Comunello, Claudia Irene Wesoloski, Roberto Cesar do Amaral e Fabio José Dallanora, publicaram conjuntamente o artigo intitulado "Acupuntura de equilíbrio como coadjuvante no tratamento de disfunções temporomandibulares" na revista científica Ação Odonto. Neste trabalho, os referidos autores também exploraram a importância da acupuntura no tratamento dessa doença que acomete diversos indivíduos na atualidade.

Matias, et al (2018) desenvolveram suas conclusões sobre a acupuntura a partir de um estudo de caso. Em uma primeira análise da paciente de 48 anos, os autores levantaram algumas informações, sendo elas:

$\mathrm{Na}$ história pregressa da paciente, notou-se o uso de medicamentos antialérgicos (Cloridrato de Ranitidina 150mg); Diclofenaco Resinato; Omeprazol de $20 \mathrm{mg}$ para o tratamento de refluxo e Citalopran $20 \mathrm{mg}$ para o tratamento da ansiedade. Nesse caso clínico, a paciente procurou o tratamento por apresentar queixa principal de dor na região da articulação temporomandibular (ATM) e porque tinha consciência de que apertava e rangia os dentes. A paciente estava com as emoções exacerbadas, constatou-se, assim, que possui problemas relacionados à ansiedade, fator este associado à sua condição bucal atual. Foi demonstrada a associação de sintomas físicos e emocionais que agem potencializando uns aos outros e que levam o paciente a uma grande perda de qualidade de vida e autoconfiança, aspectos não tão palpáveis para cirurgiões-dentistas. Ao exame clínico intraoral, observaram-se condições relativamente boas de saúde bucal necessitando apenas de raspagem e alistamento radicular em ambas as arcadas. A paciente já fazia o uso de placa miorrelaxante, a qual passou por diversos processos de reembasamento e conferência de oclusão durante as vindas da paciente para a Clínica I.

Para além do atendimento no ambiente clínico, os autores também realizaram atendimento domiciliar para conhecer o ambiente no qual a paciente está inserida, uma prática que humaniza inclusive o atendimento ao cidadão. Nessa visita, constataram que a mesma convive em um ambiente tranquilo, não apresentando nas falas e na percepção daquela realidade social conflitos aparentes, a escova de dentes estava com as cerdas em bom estado, acoplada em um local higienizado dentro do banheiro.

Após esse atendimento, em comum acordo, a paciente aceitou participar de sessões de acupuntura para melhorar sua qualidade de vida. Assim, "As sessões de acupuntura foram realizadas em horário de aula de Clínica I, realizadas pela professora Lea Maria Dallanora. Foram realizadas três sessões com duração de 30 minutos cada, sendo a última uma sessão de auriculoterapia." (Matias, et al., 2018, p. 03). Durante as sessões, a paciente não demonstrou dores, bem como não relatou no processo da acupuntura, descrevendo apenas relaxamento no processo de retirada das agulhas. Após as etapas do tratamento holístico, além da melhoria da DTM, a paciente informou ter melhorado seu sono e alívio das dores que a acometiam.

De acordo com Matias, et al. (2018, p. 05):

[...] estudos comparativos entre utilização do tradicional método da placa oclusal no tratamento da dor facial crônica resistente aos tratamentos convencionais (placa oclusal, ajuste oclusal ou exercícios físicos mandibulares) e a técnica da acupuntura mostram resultados bem semelhantes de alívio e controle da dor. 
Os autores também refletem sobre os cuidados que devem ser tomados no tratamento da DTM com a acupuntura, pois existem pessoas que são contraindicadas, como por exemplo, pessoas com marca-passo, com prótese de válvulas cardíacas, em mulheres grávidas (desde que não sejam aplicadas agulhas em locais dilatadores do colo do útero e facilitadores da contração uterina). Outrossim, Matias, et al. (2018) também são categóricos ao afirmarem que em alguns casos, a acupuntura pode ter efeitos adversos, como "[...] tontura; náusea; sangramento, comum no ato de retirada das agulhas; sonolência; condições dermatológicas; e, em alguns casos, aumento da dor nas primeiras horas após a aplicação.” Desse modo,

Para evitar problemas e tornar a prática da acupuntura segura, o acupunturista, no caso o cirurgião-dentista, deve conhecer a anatomia do corpo humano, bem como o seu funcionamento harmônico, usar agulhas descartáveis e esterilizadas, manter o paciente na posição deitada durante o tratamento, pedir ao paciente que evite dirigir após a sessão e contar as agulhas que aplicou e que retirou, para evitar o esquecimento delas no corpo. (Matias, et al., 2018, p. 07).

Entretanto, mesmo demonstrando resultados significativos, sendo de baixo custo, e eficaz na redução da dor, os autores relatam que o uso da acupuntura ainda é pouco explorado no campo da odontologia.

Outro trabalho muito importante que versa sobre a temática publicado em 2019 é dos autores Evelyn de Freitas Boscaine, Elenir Rose Jardim Cury Pontes, Daisilene Baena Castillo, Lirane da Silva Carneiro Suliano, Nelson Talatoci Oshiro Filho. Assim como a maioria dos estudos observados, trata-se de um estudo clínico que ocorreu nas dependências da Universidade do Mato Grosso do Sul. A amostra resultou em 34 voluntários portadores de DTM, diagnosticados a partir do uso do RDC. Conforme os autores "O grupo 2 foi tratado com 6 sessões de acupuntura com duração de 30 minutos cada. A dor foi avaliada pela escala analógica visual e com auxílio de um algômetro para avaliar a tensão muscular dos músculos temporal e masseter." (Boscaine, et al, 2019, p. 348). O tratamento dos pacientes foi realizado em seis semanas, com 30 minutos de duração. Após este período, "OS resultados estatísticos mostraram que a acupuntura aumentou o limiar de tensão muscular, melhorou a abertura de boca e diminuiu a dor".

Recentemente, em 2020, foi publicado o trabalho dos pesquisadores Camilla de Aguiar, Lohana Maylane de Lima, Nely Dulce Freitas, Milena Pinheiro, José Leonardo Souza, Arnaldo Caldas Júnior, Ricardo Eugenio de Melo intitulado "O uso de acupuntura e terapias integrativas no tratamento da síndrome da disfunção na articulação temporomandibular" na revista Headache Medicine. Trata-se de um estudo clínico com 50 pacientes, dos quais, 25 foram tratados com acupuntura, e 25 com métodos convencionais de tratamento da dor oriunda da DTM. Apesar de não detalharem como o processo foi realizado, número de sessões, os autores afirmaram nos resultados que "[...] a técnica da acupuntura ofereceu ao paciente uma melhor qualidade de vida, alivio das dores na ATM, mudança da qualidade da dor e diminuição de pontos-gatilhos, tudo isso em menos sessões e menor tempo de tratameto em relação as técnicas convencionais.” (Aguiar, et al, 2020, p. 1).

\section{Conclusão}

A acupuntura é uma técnica que tem se mostrado tão eficiente, quanto as terapias ocidentais, é um método simples e natural, que deve ser aliada a diversos procedimentos odontológicos. Uma perspectiva holística deve ser integrada ao tratamento, unindo todos os conhecimentos técnicos e científicos, pode proporcionar melhores resultados para o paciente, como qualidade de vida, bem-estar e saúde. Importante estabelecer que, para a medicina tradicional chinesa, o indivíduo deve ser olhado de forma holística, ou seja, portador de elementos físicos, mentais, emocionais que são indivisíveis, partes integrantes e ativas dos mais diversos ambientes (físico, natural e psicossocial).

O método da acupuntura tem a vantagem que, ao mesmo tempo em que traz o efeito analgésico, proporciona ao paciente calma e tranquilidade necessárias para identificar os fatores que possam estar desencadeando as crises. Sendo a técnica 
acessível a todas as idades e inclusiva para pacientes especiais, como trazido pelos pesquisadores, o uso dessa técnica em pacientes com síndromes e paralisias cerebrais ajuda-os a controlar movimentos involuntários, os estudos revelaram também que pacientes submetidos à acupuntura consumiram menos analgésicos e o edema facial pós-cirúrgico foi menor.

Importante destacar que, para realizar a acupuntura, o profissional deve levar em consideração as particularidades de cada paciente, explicar os efeitos positivos e aqueles colaterais, bem como não recomendar para os públicos especificados na pesquisa no tratamento da DTM. Complementarmente, o profissional precisa ter responsabilidade no processo de utilização e descarte das agulhas utilizadas, para não contaminar animas, pessoas e todo o meio ambiente.

Mesmo apresentando tantos efeitos positivos, os quais foram testados em casos reais, observa-se que a inserção da acupuntura nas clínicas odontológicas brasileiras ainda é incipiente. É necessário que as universidades instiguem os acadêmicos a desenvolverem estudos relacionados aos efeitos positivos da acupuntura não só no tratamento da DTM, para que assim seja possível estabelecer uma qualidade de vida para os pacientes, como também coloquem em prática nas clínicas universitárias, desde que acompanhados por profissionais capacitados.

\section{Referências}

Aguiar, C de. et al. (2020). O uso de acupuntura e terapias integrativas no tratamento da síndrome da disfunção na articulação temporomandibular. Headache Medicine, 11, https://headachemedicine.com.br/index.php/hm/article/view/171.

Bardin, L. (2011). Análise de conteúdo. Edições 70.

Borin, G da S. et al. (2011). Acupuntura como recurso terapêutico na dor e na gravidade da desordem temporomandibular. Fisioterapia e Pesquisa, 18(3), 217$22 \mathrm{https}: / /$ www.scielo.br/j/fp/a/G8tRHKFP6MtR8YM49vzcynH/?format=pdf\&lang=pt.

Boscaine, E de F. et al. (2019). Acupuntura no tratamento da disfunção temporomandibular muscular. BrJP. https://www.scielo.br/j/brjp/a/96pTyDGq64XQMrWZhDyTNRL/?format=pdf\&lang=pt.

Branco, C. A. et al. (2005). Acupuntura como tratamento complementar nas disfunções temporomandibulares: revisão da literatura. Revista de Odontologia da UNESP. 34(1). https://revodontolunesp.com.br/article/588017ac7f8c9d0a098b4847.

Donnarumma, M. D. C. (2010). Disfunções temporomandibulares: sinais, sintomas e abordagem multidisciplinar. Rev. CEFAC. https://www.scielo.br/j/rcefac/a/rd7PJ8RQW3KvYSkPsw9gxJd/?lang=pt\&format=pdf.

Ferreira, L A. et al. (2015). Ear Acupuncture Therapy for Masticatory Myofascial and Temporomandibular Pain: A Controlled Clinical Trial. Hindawi Publishing Corporation, https://www.hindawi.com/journals/ecam/2015/342507/.

Florian, M R F. Meirelles, M P M R., Sousa, M da L R de S. (2011). Disfunção temporomandibular e acupuntura: uma terapia integrativa e complementar. Odontol. Clín.Cient., 10 (2) 189-192, http://revodonto.bvsalud.org/pdf/occ/v10n2/a19v10n2.pdf.

fonseca, D Ms da. et al. (1994). Diagnóstico pela anamnese da disfunção craniomandibular. Rev. Gaúcha Odontol, 42, https://pesquisa.bvsalud.org/portal/resource/pt/biblio-854978.

Freire, J C P., Freire, S C P., \& Dias-Ribeiro, E. (2018). Análise da acupuntura no tratamento de dores orofaciais: estudo de casos. Rev. Odontol. Univ. São Paulo, 30(1): 16-20.

Garbelotti, T O. et al. (2016). Effectiveness of acupuncture for temporomandibular disorders and associated symptoms. Rev Dor. São Paulo, https://www.scielo.br/j/rdor/a/qLJKt39L7JBNMpQRzLTmLLJ/?format=pdf\&lang=pt.

Gil, A C. (2008). Como elaborar projetos de pesquisa. (5a ed.), Atlas.

Hideaki, O. et al. Effects of trigger point acupuncture treatment on temporomandibular disorders: a preliminary randomized clinical trial. Journal of Acupuncture and Meridian Studies, 5(2), 57-62, 2012. https://pubmed.ncbi.nlm.nih.gov/22483183/.

Lakatos, E. M., \& Marconi, M de A. (2011). Fundamentos de metodologia científica. (9a ed.), Atlas.

Matias, B J. et al. Acupuntura de equilíbrio como coadjuvante no tratamento de disfunções temporomandibulares. Ação Odonto. 2018. https://portalperiodicos.unoesc.edu.br/acaodonto/article/view/17191.

Neville, B. W. et al. Patologia oral e maxilofacial. (4a ed.), Elsevier, 2016.

Pereira Júnior, F. J., \& Gonçalves, D. A. G. Diagnostic criteria for temporomandibular disorders: assessment instruments. Ed. Richard Ohrbach, 2014.

Schiffman, E. et al. Diagnostic Criteria for Temporomandibular Disorders (DC/TMD)

for Clinical and Research Applications: Recommendations of the International RDC/TMD Consortium Network and Orofacial Pain Special Interest Group. J Oral Facial Pain Headache, 2014. 
Research, Society and Development, v. 11, n. 1, e31911125052, 2022

(CC BY 4.0) | ISSN 2525-3409 | DOI: http://dx.doi.org/10.33448/rsd-v11i1.25052

Yamamura, Y. (2004). Acupuntura tradicional: a arte de inserir. (2a ed.), Roca.

Zotelli, V L R., Meirelles, M P M R., Sousa, M da L R de. Uso da acupuntura no manejo da dor em pacientes com alterações na articulação temporomandibular (ATM). Revista de Odontologia da Universidade Cidade de São Paulo, 22, https://publicacoes.unicid.edu.br/index.php/revistadaodontologia/article/view/415.

Zotelli, V L R. et al. Acupuncture effect on pain, mouth opening limitation and on the energy meridians in patients with temporomandibular dysfunction: a randomized controlled trial. Journal of Acupuncture and Meridian Studies, 2017. https://pubmed.ncbi.nlm.nih.gov/29078971/. Acesso em: 21 nov. 2021. 